University of Nebraska - Lincoln

DigitalCommons@University of Nebraska - Lincoln

USDA National Wildlife Research Center - Staff Publications
U.S. Department of Agriculture: Animal and Plant Health Inspection Service

2010

\title{
Response of Captive Skunks to Microencapsulated Tetracycline
}

\author{
Brandon S. Schmit \\ USDA/APHIS/WS National Wildlife Research Center, Brandon.S.Schmit@aphis.usda.gov \\ Thomas M. Primus \\ United States Department of Agriculture \\ Jerome C. Hurley \\ United States Department of Agriculture \\ Dennis J. Kohler \\ USDA/APHIS/WS National Wildlife Research Center, dennis.kohler@aphis.usda.gov \\ Shawna F. Graves \\ United States Department of Agriculture
}

Follow this and additional works at: https://digitalcommons.unl.edu/icwdm_usdanwrc

Part of the Environmental Sciences Commons

Schmit, Brandon S.; Primus, Thomas M.; Hurley, Jerome C.; Kohler, Dennis J.; and Graves, Shawna F., "Response of Captive Skunks to Microencapsulated Tetracycline" (2010). USDA National Wildlife Research Center - Staff Publications. 1064.

https://digitalcommons.unl.edu/icwdm_usdanwrc/1064

This Article is brought to you for free and open access by the U.S. Department of Agriculture: Animal and Plant Health Inspection Service at DigitalCommons@University of Nebraska - Lincoln. It has been accepted for inclusion in USDA National Wildlife Research Center - Staff Publications by an authorized administrator of DigitalCommons@University of Nebraska - Lincoln. 


\title{
Response of Captive Skunks to Microencapsulated Tetracycline
}

\begin{abstract}
Brandon S. Schmit, ${ }^{1,2,4}$ Thomas M. Primus, ${ }^{1,3}$ Jerome C. Hurley, ${ }^{1}$ Dennis J. Kohler, ${ }^{1,2}$ and Shawna F. Graves ${ }^{1}{ }^{1}$ National Wildlife Research Center, United States Department of Agriculture, Animal and Plant Health Inspection Service, Wildlife Services, Fort Collins, Colorado 80521-2154, USA; ${ }^{2}$ Current address: National Wildlife Disease Program, United States Department of Agriculture, Animal and Plant Health Inspection Service, Wildlife Services, Fort Collins, Colorado 80521-2154, USA; ${ }^{3}$ Deceased; ${ }^{4}$ Corresponding author (email: brandon.b.schmit@aphis.usda.gov)
\end{abstract}

ABSTRACT: A captive striped skunk (Mephitis mephitis) study was conducted between February and June 2004 at the United States Department of Agriculture, Animal and Plant Health Inspection Service, Wildlife Services National Wildlife Research Center, Fort Collins, Colorado, USA. The main objective was to determine the percentage of adult striped skunks that were marked after consuming placebo oral rabies vaccine (ORV) baits containing $100 \mathrm{mg}$ of an experimental microencapsulated (coated microparticle) tetracycline hydrochloride biomarker. Biomarkers were identified in the canine teeth and mandibles of five of five skunks that consumed an ORV bait. A second objective was to determine if the microencapsulated tetracycline was resistant to photochemical conversion from tetracycline to epitetracycline. After 15 days of exposure, conversion from tetracycline to epitetracycline concentration in the microencapsulated product (mean $1.9 \%$ conversion, $\mathrm{SD}=1.24$ ) was significantly less $(P=0.006)$ than the pure-grade tetracycline powder (mean $7.5 \%$ conversion, $\mathrm{SD}=1.37$ ). Results support the use of microencapsulated tetracycline hydrochloride as a biomarker in circumstances where the use of conventional powdered tetracycline hydrochloride is not feasible due to ORV bait design constraints.

Key words: Coated sachet, Mephitis mephitis, oral rabies vaccination, pen study, striped skunk, tetracycline biomarker.

Oral rabies vaccine (ORV) baits typically contain tetracycline hydrochloride as a biomarker (Johnston and Tinline, 2002). The tetracycline biomarker becomes incorporated into the teeth and bones of animals that consume the ORV baits (Johnston et al., 1999). These permanent marks in the teeth allow ORV program coordinators to evaluate baiting success by trapping animals within an ORV zone, extracting a tooth from each individual for tetracycline analysis, and determining the number of target animals that have consumed one or more baits. Discrepancies between biomarker and vaccination levels in the target population allow ORV programs to discover potential problems with a particular vaccine lot, or the ORV bait itself. Biomarker data can also be used to optimize ORV program strategies by comparing bait consumption in the target species to variation in baiting density and flight line intervals (Johnston and Tinline, 2002).

The United States Department of Agriculture (USDA), Animal and Plant Health Inspection Service (APHIS), Wildlife Services (WS) ORV Program currently uses an extruded fish-meal polymer bait (FMP; MERIAL Ltd., Athens, Georgia, USA) that contains $150 \mathrm{mg}$ of tetracycline hydrochloride as a biomarker (Slate et al., 2005). The relatively large volume of the FMP allows for the tetracycline biomarker to be added as a powder to the bait material before extrusion. This allows for homogeneous mixing of the biomarker throughout the bait (Johnston et al., 2005) and may mask or dilute the bitter taste (del Castillo et al., 2002) of the tetracycline hydrochloride powder. MERIAL Ltd. also manufactures a coated sachet (CS) bait that utilizes the same vaccine sachet as the FMP, but has a minimal fish-meal coating that does not contain a biomarker. The CS bait has shown promise in raccoons (Procyon lotor) and coyotes (Canis latrans; Linhart et al., 2002), in captive bait acceptance trials in skunks (Mephitis mephitis; Jojola et al., 2007), and is currently used in the WS ORV Program as an alternative to the FMP bait for vaccinating raccoons. Unfortunately, skunks have proven difficult to 
vaccinate, as indicated by poor seroconversion rates within ORV zones (Rosatte, 2008). Grosenbaugh et al. (2007) found that four of four of skunks that seroconverted after direct oral installation of Raboral V-RG ${ }^{\circledR}$ survived a rabies challenge. Ten skunks in the same study did not develop rabies virus-neutralizing antibodies after consumption of either one or three CS baits containing Raboral V$\mathrm{RG}^{\circledR}$, and nine of these ten skunks died upon rabies challenge. These results suggest that the CS bait is not delivering the vaccine to striped skunks effectively during consumption, and that improved ORV baits need to be developed for use in skunks. Rosatte et al. (2008) attributed these low seroconversion rates skunks in Canada to CS baits having been designed for raccoons and other larger animals. Jojola et al. (2004) suggest that smaller baits would allow skunks to puncture the vaccine container more easily.

Smaller ORV baits intended for skunks would still require adequate tetracycline biomarker concentrations (>80 mg). The higher ratio of tetracycline to bait material on these improved smaller bait designs may result in palatability issues if tetracycline hydrochloride powder is used. In an effort to improve palatability and field stability of the tetracycline biomarker, an experimental microencapsulated tetracycline was developed at the USDA APHIS WS National Wildlife Research Center (NWRC), Fort Collins, Colorado, USA. The microencapsulation process coats small particles of tetracycline with a polymer and results in a tasteless granular product, similar to sustained-release pharmaceutical products.

To produce the microencapsulated tetracycline used in this study, tetracycline hydrochloride $(500 \mathrm{~g})$ and Avicel 101 (100 g; microcrystalline cellulose tableting material; FMC Corporation, Philadelphia, Pennsylvania, USA) were placed in a Kitchen-Aid mixing bowl and $30 \mathrm{~g}$ of Kollidon 30 (a binding agent; BASF, Florham Park, New Jersey, USA) in
$300 \mathrm{mdl}$ of water was slowly added with mixing. The wetted mass was pushed through a No. 7 sieve and the pieces were dried in a $60-\mathrm{C}$ oven to $1-3 \%$ moisture. This material was force sieved through a series of sieves and the fraction of particles smaller than No. 20 and greater than No. 40 sieves in size were retained. The remaining particles were reworked through the wet sieve granulation steps until all were of the same approximate size. Based on the particle size $(\sim 0.8 \mathrm{~mm})$ and a desired coating level of $0.2 \mathrm{mg} / \mathrm{cm}^{2}$, a coating level of $20 \%$ was determined. The coating consisted of EPO (an aminesubstituted polyacrylate; Rohm Pharma Polymers, Piscataway, New Jersey, USA), acetyltriethyl citrate (coating plasticizer; Morflex Inc., Greensboro, NC, USA) and sodium dodecyl sulfate (surfactant; Sigma Chemical Co., St. Louis, Missouri, USA) dispersed in water. Using a fluidizing bed coating machine (Strea-1; AeromaticFielder, Eastleigh, Hampshire, UK), the coating suspension was sprayed onto the particles with an inlet temperature of $40 \mathrm{C}$, outlet temperature of $28 \mathrm{C}$, and an application rate of approximately $5 \mathrm{ml} / \mathrm{min}$. The resulting microencapsulated tetracycline concentration was approximately $72 \%$ tetracycline by weight $(68 \%$ tetracycline and $4 \%$ epitetracycline).

To test the microencapsulated tetracycline's ability to prevent photoconversion of tetracycline to epitetracycline, three $1-g$ replicates of the product and three 1-g replicates of a technical-grade tetracycline powder were placed in an environmental chamber for 15 days. The environmental chamber conditions were set for periods of $12 \mathrm{hr}$ of light and $12 \mathrm{hr}$ of dark per day. The light period conditions averaged $28 \mathrm{C}$ with an average relative humidity of $55 \%$. The dark period conditions averaged $20 \mathrm{C}$ with an average relative humidity of $65 \%$. The radiant energy of the daylight period was monitored with a radiometer (UVX Digital Radiometer, UVP, Inc, Upland, California, USA) with three different sensors. The three sensors (R-25 $254 \mathrm{~nm}$, 
R-31 $310 \mathrm{~nm}$, and R-36 $365 \mathrm{~nm}$ ) averaged $11.5,27.0$, and $52 \mu \mathrm{W} / \mathrm{cm}^{2}$. The same three sensors measured 8.6, 32.6, and $21.5 \mu \mathrm{W} / \mathrm{cm}^{2}$ respectively in natural light during a cloudy day on 25 May 2005 at the NWRC. The tetracycline concentration of the microencapsulated product versus the technical-grade tetracycline was analyzed by removing three $5-\mathrm{mg}$ samples every third day from the environmental chamber. All samples were immediately analyzed according to high-performance liquid chromatography protocols established for tetracycline analysis at the NWRC (Primus et al., 2005). All percent tetracycline values for each sample were arc sine transformed to allow student $t$-test comparisons between microencapsulated and tech-grade tetracycline replicates after environmental chamber exposure. Student's $t$-test was performed with the use of the TTEST formula in Excel 2007.

Experimental placebo CS baits were produced by mixing the microencapsulated tetracycline with the standard fish-meal coating bait material. This mixture was then applied to the exterior of the sachet so that the resulting CS bait contained $100 \mathrm{mg}(80 \mathrm{mg}$ of tetracycline $+20 \mathrm{mg}$ coating and clumping materials) of the microencapsulated product. There were no observed differences in the durability of the coating of regular sachets versus sachets with the added microencapsulated tetracycline. A second bait type, the Artemis (Artemis Technologies, Inc. Guelph, Ontario, Canada) blister packs (AB) were prepared in the same manner.

Twenty individually housed skunks were retained from a previous study between February and June 2004 and were fed a diet consisting of $100 \mathrm{~g}$ Mazuri Omnivore Zoo Feed "A" daily and water ad libitum. Ten skunks were given the opportunity to consume a single placebo CS bait containing $100 \mathrm{mg}$ of the microencapsulated tetracycline on its exterior. Ten additional skunks were given the opportunity to consume a single placebo Artemis blister pack to which fish-meal crumbles and 100-mg microencapsulated tetracycline had been added. Each skunk was given a single bait to consume on a given night. One hour after sunset, the bait was placed in the center of a plate and the next morning any remaining parts of the bait were examined. All baits included $80 \mathrm{mg}$ of active tetracycline, which is at least two times the amount of tetracycline needed to biomark an average-sized $(4 \mathrm{~kg})$ adult striped skunk (10 mg tetracycline $/ \mathrm{kg}$ body weight; Johnston et al., 1999). Therefore, any bait remnant that was visually observed to be missing over $50 \%$ of the bait coating was classified as a potentially successful biomarking. Each skunk was allowed to consume only one bait. All skunks that successfully consumed a bait remained on test for a minimum of 8 wk to allow the tetracycline biomarker, if present, to be more easily discerned in the teeth and jaw tissues. All skunks were then euthanized and a transverse section was taken through the mandible and canine tooth root for biomarker analysis. The tetracycline biomarker analyses were performed at the NWRC according to established protocols (Johnston and Watt, 1981; Johnston et al., 1999).

Epitetracycline formation during the extrusion process of the FMP bait has been shown to result in a decrease in the bait's marking potential (Johnston et al., 2005). To maintain optimum biomarking efficiency, ORV bait designs should minimize conversion of tetracycline to epitetracycline both during the manufacturing process as well as environmental exposure when distributed in the field. After 15 days of exposure, conversion from tetracycline to epitetracycline concentration in the microencapsulated product (mean 1.9\% conversion, $\mathrm{SD}=1.24$ ) was significantly less $(P=0.006)$ than the pure-grade tetracycline powder (mean $7.5 \%$ conversion, $\mathrm{SD}=1.37$ ). The microencapsulated material affords substantial protection from photochemical conversion of tetracycline to epitetracycline in a simulated natural light environment. 
The main objective of this study was to determine the efficacy of the experimental microencapsulated tetracycline in biomarking captive adult striped skunks. Five of 20 skunks successfully consumed an ORV bait before the study was concluded. The $25 \%$ bait acceptance rate may have been influenced by the fact that these skunks had been in captivity for 4-6 mo before the experiment began and were well fed and accustomed to the captive diet. These five individuals ranged between 3.2 and $5.3 \mathrm{~kg}$ in body weight. One male and one female skunk successfully consumed $100 \%$ of the CS bait and two male skunks consumed $75 \%$ and $50 \%$ of their CS baits, respectively. Only one female skunk consumed $75 \%$ of an $\mathrm{AB}$ bait. All five of the skunks were positive for the tetracycline biomarker in the canine tooth cementum layer and surrounding mandibular bone tissue.

There were no apparent differences in the strength of the tetracycline signatures in the teeth and bone tissues, despite the differences in the amount of bait and biomarker consumed and the weights of the skunks as confounding factors. These results suggest that $100 \mathrm{mg}$ of the microencapsulated tetracycline per bait is sufficient to mark adult striped skunks of both sexes in a field setting. Ideally, a single ORV bait would be efficacious in all target species. If such a bait were designed with the use of this microencapsulated tetracycline, controlled pen studies would be warranted to examine the biomarking capability of this microencapsulated tetracycline hydrochloride in all age classes and species of skunks as well as other ORV target species such as raccoons, coyotes, and gray foxes (Urocyon cinereoargenteus). There is supporting evidence that the microencapsulated tetracycline used in this study will biomark raccoons in a field setting. A study conducted by the first author in Louisiana in which CS baits containing $100 \mathrm{mg}$ microencapsulated tetracycline were distributed into the environment at a density of 75 baits $/ \mathrm{km}^{2}$ indicated that $57 \%$ (eight of 14) of raccoons captured were positive for the tetracycline biomarker. The number of individual raccoons that actually consumed a bait and the background levels of fluorescence in this population is unknown, however.

The authors thank Dr. Gordon Gathright and the NWRC animal care staff for their support during the study, Merial Limited and Artemis Technologies, Inc. for providing bait materials, Susan Jojola for providing the skunks, and Jeff Root for critical review of early drafts of this manuscript.

\section{LITERATURE CITED}

del Castillo, J. R. E., J. Elsener, and G. P. Martineau. 1998. Pharmacokinetic modeling of in-feed tetracyclines in pigs using a metaanalytic compartmental approach. Journal of Swine Health and Production 6: 189-202.

Grosenbaugh, D., J. Maki, C. Rupprecht, and D. WALL. 2007. Rabies challenge of captive striped skunks (Mephitis mephitis) following oral administration of a live vaccinia-vectored rabies vaccine. Journal of Wildlife Diseases 43: 124128.

Johnston, D. H., And R. R. Tinline. 2002. Rabies control in wildlife. In Rabies, A. C. Jackson and W. H. Wunner (eds.). Academic Press, San Diego, California, pp. 445-471.

$\longrightarrow$, AND I. D. WATT. 1981. A rapid method for sectioning undecalcified carnivore teeth for aging. In World Furbearer Conference Proceedings, J. A. Chapman and D. Pursley (eds.). Frostburg, Maryland. 1: 407-422.

, D. G. Joachim, P. Bachman, K. V. Kardong, R. E. A. Stewart, L. M. Dix, M. A. Strickland, AND I. D. WATt. 1999. Aging furbearers using tooth structure and biomarkers. In Wild furbearer management and conservation in North America, M. Novak, J. A. Baker, M. E. Obbard and B. Malloch (eds.). Ontario Ministry of Natural Resources, Ottawa, Ontario, Canada, pp. 228-243.

Johnston, J. J., T. M. Primus, T. Buettgenbach, C. A. Furcolow, M. J. Goodall, D. Slate, R. B. Chipman, J. L. Snow, and T. J. Deliberto. 2005. Evaluation and significance of tetracycline stability in rabies vaccine baits. Journal of Wildlife Diseases 41: 549-558.

Jojola, S. M., S. J. Robinson, and K. C. VercauteREN. 2007. Oral rabies vaccine (ORV) bait uptake by captive striped skunks. Journal of Wildlife Diseases 43: 97-106. 
Linhart, S. B., J. C. Whodkowski, D. M. Kavahaugh, L. Motes-Kreimeyer, A. J. Montoney, R. B. Chipman, D. Slate, L. L. Bigler, and M. G. Fearneyhough. 2002. A new flavor-coated sachet bait for delivering oral rabies vaccine to raccoons and coyotes. Journal of Wildlife Diseases 38: 363-377.

Primus, T., S. F. Graves, and D. J. Kohler. 2005. Assay of tetracycline and epitetracycline in encapsulated product. Analytical Chemistry Project report to Brandon Schmit, Wildlife Disease Program, USDA APHIS Wildlife Services National Wildlife Research Center, Fort Collins, Colorado.

Rosatte, R., M. Allan, P. Bachmann, K. Sobey, D. Donovan, J. C. Davies, A. Silver, K. Bennett, L. Brown, B. Stevenson, T. Buchanan, L.
Bruce, A. Wandeler, C. Fehlner-Gardiner, A. Beresford, A. Beath, M. Escobar, J. Maki, And C. Schumacher. 2008. Prevalence of tetracycline and rabies virus antibody in raccoons, skunks, and foxes following aerial distribution of V-RG baits to control raccoon rabies in Ontario, Canada. Journal of Wildlife Diseases 44: 946964.

Slate, D., C. E. Rupprecht, J. A. Rooney, D. Donovan, D. H. Lein, and R. B. Chipman. 2005. Status of oral rabies vaccination in wild carnivores in the United States. Virus Research 111: 68-76.

Submitted for publication 14 November 2008.

Accepted 25 February 2010. 